\title{
Die Selbsterregung von Kommutatormotoren während ihres Laufes als Motor.
}

\author{
Von
}

\section{Schenkel.}

1. Die Zuordnung von Fluß und MMK des Ankers zur Drehrichtung beim Motor und Generator. Wenn eine Maschine elektrische Arbeit in mechanische umsetzen oder umgekehrt mechanische Arbeit in elektrische verwandeln soll, so müssen die Flüsse, die stromführenden Ankerleiter und die Drehrichtung nach bestimmten Regeln im Raume angeordnet sein. Diese sind durch das Induktionsgesetz und das elektrodynamische Kräftegesetz bestimmt und ergeben ein sehr einfaches Unterscheidungsmerkmal dafür, ob die Maschine als Motor oder Generator arbeitet.

In Fig. I und 2 ist durch den nach rechts gerichteten Pfeil $\Phi$ die Raumrichtung eines Flusses $\Phi$ festgelegt. In ihm läuft im Uhrzeigersinne ein Anker um, dessen Leiter Strom führen. Die Stromverteilung auf den Umfang dieses Ankers sei so eingerichtet, daß in Fig. I alle Leiter, die sich rechts von einer zur $\Phi$-Richtung senkrechten Ebene befinden.

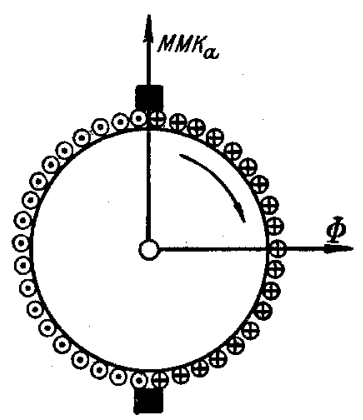

Fig. I.

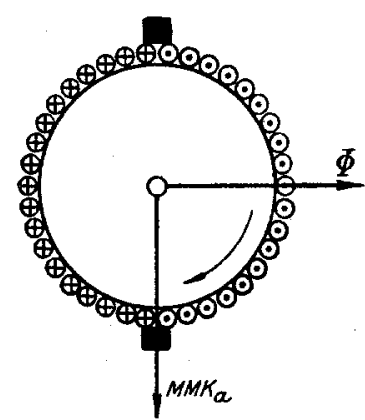

Fig. 2 .

nach hinten gerichtete Ströme führen. Alle Leiter links der Ebene sollen nach vorn gerichtete Ströme haben. In Fig. 2 sei die Stromverteilung gerade umgekehrt: rechts die nach vorn, links die nach hinten gerichteten Ströme. Fig. I entspricht einem Motor, Fig. 2 einem Generator

Unter diesen Voraussetzungen läßt sich die ganze magnetomotorische Kraft des Ankers durch einen Pfeil MMKa darstellen, der in Fig. I nach oben, in Fig. 2 nach unten zeigt.

Damit erhalten wir folgendes Unterscheidungszeichen:

„Die Drehung beim Motor führt von der räumlich positiven Richtung der AnkerMMK nach der räumlich positiven Flußrichtung hin."

„Die Drehung beim Generator führt von der räumlich positiven Flußrichtung nach der räumlich positiven Richtung der Anker-MMK hin."

Dieses Merkmal muß für jede Maschine gelten, und zwar für jedes Polpaar, das sie hat. Es muß auch gelten für jeden Strom irgendeiner Periodenzahl, der sich in der Maschine findet.

MMKa und $\Phi$ brauchen nicht senkrecht zueinander $\mathrm{zu}$ stehen. Bilden beide einen spitzen oder stumpfen Winkel, so gilt die auf einem der beiden Vektoren senkrecht stehende Komponente des anderen. Das gleiche gilt von einer zufällig vorhandenen zeitlichen Phasenverschiebung.

2. Die Übereinanderlagerung einer generatorischen Wirkung über eine motorische. Es sei eine Maschine als Motor im Betriebe. Der ihr zugeführte Strom bringt also 
in der Maschine einen Fluß $\Phi$ und in dem Anker eine magnetomotorische Kraft MMKa hervor, die so gerichtet sind, wie Fig. I anzeigt.

Soll diese Maschine zugleich Selbsterregungserscheinungen zeigen, so müssen die Selbsterregungsströme solche Fluß- und MMK-Verteilungen hervorbringen, wie es Fig. 2 angibt. Denn Selbsterregung ist gleichbedeutend mit generatorischer Wirkung. Diese neuen generatorischen Flüsse und magnetomotorischen Kräfte müssen den motorischen der Fig. I überlagert sein.

Die allgemeine Lösung dieser Aufgabe würde darin bestehen, Spannungsgleichungen für die vorhandenen Stromkreise aufzustellen, wie dies bereits a. a. $\mathrm{O}^{1}$ ) getan worden ist, und diesen noch die Bedingung hinzuzufügen, daß die von außen zugeführten Ströme mechanische Arbeit leisten.

Im folgenden sollen an zwei Motoren, einem einphasigen und einem dreiphasigen, zwei spezielle Lösungen dieser Aufgabe näher beschrieben werden.

Da an der Erzeugung der generatorischen und motorischen Wirkung immer der Anker beteiligt sein muß, so soll in beiden Fällen zuerst dieser betrachtet werden, jedoch nur in der Ausführung mit Durchmesserwicklung. Anker mit Sehnenwicklung können andere Ergebnisse bringen, aber sie werden - außer solchen mit fast durchmessergleichen Sehnen - sehr selten benutzt. Wirkungen von Unsymmetrien im Bau der Maschine seien ausgeschlossen.

3. Selbsterregung bei einem doppelt gespeisten Reihenschlußmotor. Beispiel I. In einem Anker, der über 2 Bürsten gespeist wird, fließe außer dem zugeführten Strome noch ein zweiter überlagerter.

Dieser Strom muß sich, damit ein ihm entsprechender Fluß entsteht, in die andern Wicklungen der Maschine ausbreiten. Dies ist nur möglich, wenn er den Anker auf dieselbe Weise betritt und verläßt wie der motorisch wirkende Strom. Der überlagerte Strom und sein zugeordneter Fluß könnten also nur in Frequenz und Richtung vom motorischen Strom verschieden sein, während die ihnen zugehörige Polzahl mit der des motorischen Stroms übereinstimmen muß.

Ein derartiger Fall ist für die in Fig. 3 dargestellte Schaltung bekannt geworden ${ }^{2}$ ). Sie stellt einen einphasigen, doppelt gespeisten Motor dar, der durch Bürstenverschiebung geregelt und aus einem Transformator gespeist wird, von dem in der Figur nur die sekundären Wicklungen zu sehen sind. Die Wicklung I speist den Anker A, die Wicklung II den Stator S. Die Richtung, in der eine der 4 Wicklungen im positiven Sinne durchlaufen wird, geht stets von a (Anfang) nach e (Ende). Denkt man sich die doppelte Speisung durch Öffnung des Schalters $\mathrm{H}$ aufgehoben, so hat man einen einfachen Reihenschlußmotor ohne Erregerwicklung vor sich und sieht, daß die beiden Transformatorwicklungen,

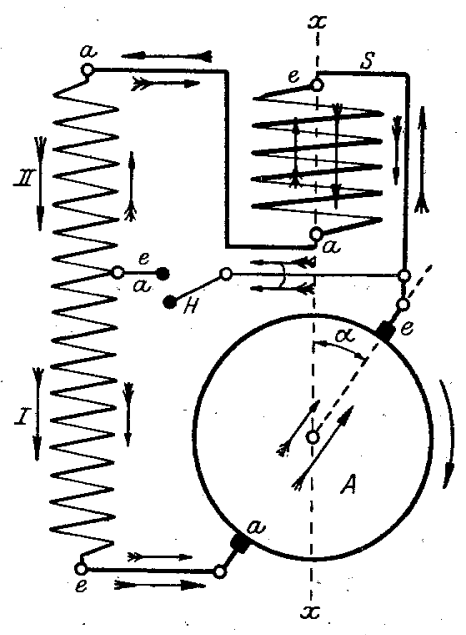

Fig. 3 . sich unterstützend, auf den Motoranker A wirken, der durch die im Sinne e-a angeschlossene Statorwicklung S kompensiert wird. Infolge der Bürstenverschiebung $\alpha$ erregt, läuft der Anker gemäß Fig. I im Sinne des Rundpfeils um. Der vom Netz gelieferte Strom ist durch große Pfeile mit einer Feder angegeben.

Wird der Schalter $\mathrm{H}$ geschlossen, dann ist ein Selbsterregungsstrom möglich, der in einem Moment so fließen kann, wie es die kleineren Pfeile mit doppelter Feder angeben.

1) Archiv der Elektrotechnik I9I2, Bd. I, S. 34. Artikel von R, Rùdenberg über „Elektrische Eigenschwingungen in Dynamomaschinen".

2) Siehe EKB. rgır, S. 726. Aufsatz von P. Müller. 
Wie ohne weiteres an Hand dieser Pfeile erkennbar ist, besitzen die räumlichen Richtungen des resultierenden Flusses und der magnetomotorischen Kraft des Ankers Lagen zueinander, wie sie gemäß Fig. 2 bei einem Generator vorkommen, mit dem einzigen Unterschiede, daß der Winkel zwischen ihnen spitz ist, während er in Fig. $290^{\circ}$ beträgt. Die motorische Kraft des Netzstromes wird also durch die generatorische Wirkung des Selbsterregungsstromes teilweise aufgezehrt, der Motor bremst sich selbst ab.

Die Möglichkeit dieser Selbsterregung während des Motorbetriebes läßt sich am kürzesten so beweisen, wie es Rüdenberg in der Arbeit ,Elektrische Eigenschwingungen in Dynamomaschinen" im Archiv für Elektrotechnik, I. Band, I9I2, r. Heft, S. 36, ermittelt hat. Es ist dort gezeigt worden, daB 2 Stromsysteme I und 2 stationäre Schwingungen haben, wenn ihre Wechselinduktivitäten $\mathrm{M}_{1}$ und $\mathrm{M}_{2}$, die sie infolge ruhender Transformation besitzen und ihre Rotations-Induktivitäten $\mathrm{N}_{1}$ und $\mathrm{N}_{2}$, die sie infolge der Bewegung von Ankern mit Kollektoren besitzen, der Bedingung genügen

$$
\mathrm{M}_{1} \cdot \mathrm{N}_{2}+\mathrm{M}_{2} \cdot \mathrm{N}_{1}=\mathrm{K} \text {, }
$$

worin $\mathrm{K}$ eine stets positive Größe ist, die sich aus Widerständen und Eigeninduktivitäten zusammensetzt, die wir aber für den vorliegenden Fall nicht näher zu untersuchen brauchen.

Für unsern Motor ist $\mathrm{N}_{2}=\mathrm{O}$, da der Kreis 2 - Wicklung II und Stator $\mathrm{S}$ - keinen Anker mit Kollektor enthält.

$\mathrm{M}_{2}$ setzt sich aus 2 Wechselinduktivitäten zusammen, nämlich der des Transformators $M_{\tau}$ und der des Stators, die wegen der veränderlichen, von der magnetischen Achse $\mathrm{x}-\mathrm{x}$ gerechneten Bürstenverschiebung $\alpha$ den Wert $\mathrm{M} \cdot \cos \alpha$ besitzt. Da der Stator $\mathrm{S}$ im negativen Sinne geschaltet ist, so $\mathrm{mu} ß \mathrm{M} \cdot \cos \alpha$ mit negativem Vorzeichen erscheinen, so daß man hat

$$
\mathrm{M}_{2}=\mathrm{M}_{\tau}-\mathrm{M} \cdot \cos \alpha .
$$

Auch $N_{1}$ ist mit $\alpha$ veränderlich und erscheint wegen der negativen Schaltung von $S$ mit negativem Zeichen, mithin ist

$$
\mathrm{N}_{1}=-\mathrm{N} \cdot \sin \alpha
$$

Man erhält also

$$
\mathrm{M}_{2} \cdot \mathrm{N}_{1}=-\left(\mathrm{M}_{\tau}-\mathrm{M} \cdot \cos \alpha\right) \cdot \mathrm{N} \cdot \sin \alpha=\mathrm{K} \text {. }
$$

Der Einfachheit wegen betrachten wir nur das Gebiet von $\alpha=-90^{\circ}$ bis $\alpha=+90^{0}$, das dem Motorbetriebe nach beiden Drehrichtungen am meisten entspricht.

In ihm ist $\cos \alpha$ immer positiv. Ist $\alpha$ positiv, so braucht nur $M \cdot \cos \alpha>M_{\tau}$ zu sein, ist $\alpha$ negativ, so braucht nur $M \cdot \cos \alpha<M_{\tau}$ zu sein, und es tritt Selbsterregung ein. Der letzte Fall ist sehr leicht erfüllt, da $M_{\tau}$ wegen des völligen Eisenschlusses im Transformator zumeist größer ist als das $M$ des Motors, der ohne einen Luftspalt nicht ausführbar ist. Da mit dem Vorzeichen von $\alpha$ zugleich die Motordrehrichtung wechselt, wird also die Maschine bei einer Drehrichtung Selbsterregung zeigen.

Diese Neigung zur Selbsterregung ist als ein Nachteil der sonst sehr zweckmäßigen Maschine anzusehen. Die Selbsterregung tritt plötzlich und mit großer Gewalt auf, wenn nicht Dämpfungswiderstände in den Stromkreisen liegen. Diese verzehren aber wiederum einen Teil der Netzleistung, so daß der Wirkungsgrad der Maschine sinkt. Sättigung und eigene Widerstände werden innerhalb eines gewissen Arbeitsgebietes wohl keine Selbsterregung aufkommen lassen; es ist indessen noch nicht bekannt geworden, wie weit sie genügen, um den Betrieb der Maschine zu gestatten. Die erwähnte Literaturstelle zeigt, daß man bei ausgeführten Maschinen dieser Art mit Selbsterregungen störender Natur zu kämpfen hatte.

4. Selbsterregung bei einem Drehstrom-Reihenschluss-Motor. Beispiel 2. Während im ersten Beispiel der Selbsterregungsstrom, von seiner augenblicklichen Richtung abgesehen, die vorhandenen Wicklungen in ähnlicher Weise durchfließt wie der Netzstrom und infolge der Speisung des Ankers an 2 Punkten ebensoviel Pole ausbildet 
wie der Netzstrom, kann eine Speisung des Ankers an mehreren Punkten ganz anders verlaufende Selbsterregungsströme und die Ausbildung ganz anderer Polzahlen zur Folge haben

Wir werden den Fall betrachten, daß der Anker an sechs Punkten gespeist wird, ein Fall, der bei den neueren Drehstromkollektormotoren oft vorkommt.

Besitzt der Anker, wie vorausgesetzt, eine Durchmesserwicklung, so müssen immer diametral gegenüberliegende Leiter verschiedene Stromrichtung haben. Wie Fig. 4 veranschaulicht, können $\mathrm{n}$ um den Anker symmetrisch herumgelagerte Polpaare entstehen, wenn die Zahl n der Bürstenpaare ungerade ist. Der an sechs Punkten gespeiste Anker vermag also trotz seiner für 2 Pole eingerichteten Durchmesserwicklung 3 Polpaare oder 6 Pole zu erzeugen, wenn der Strom in die Bürsten I, 3 und 5 ein- und bei den Bürsten 2, 4 und 6 austritt. Dieser Stromverlauf kann sich

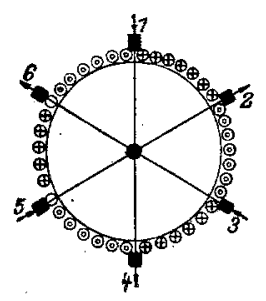

Fig. 4. unter Umständen beim Motorbetriebe eines an sich zweipolig gebauten Motors tatsächlich von selbst aufrecht erhalten.

Wir betrachten einen Drehstrom-Reihenschlußmotor, der ebenfalls wie unser erstes Beispiel mit Bürstenverschiebung geregelt wird.

a) Die Wicklung des DrehstromReihenschlußmotors. Fig. 5 zeigt diesen Motor mit den Bürsten in der - betriebsmäßig

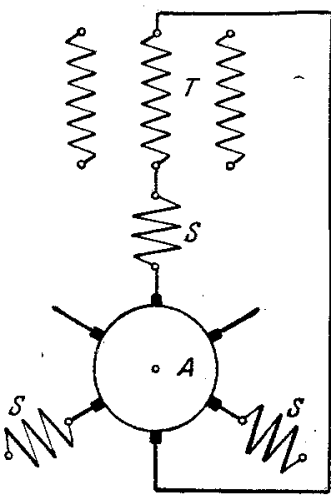

Fig. 5 nicht vorkommenden - Kurzschlußstellung, in der er kein Drehmoment entwickelt, sondern dem Netz einen starken Kurzschlußstrom entnimmt. Jede Phase wird aus einer Sekundärwicklung eines dreiphasigen Transformators $\mathrm{T}$ gespeist. In der Figur ist nur der Anschluß einer Phase gezeichnet, um die Übersichtlichkeit zu wahren. Die Primärwicklung des Transformators $T$ ist nicht gezeichnet. S ist die Ständerwicklung, A der Anker.

Wir bringen das Bild dieser betriebsmäßig gar nicht brauchbaren Stellung der Bürsten, um daran die Wicklung zu erläutern, die ein solcher Motor haben mu richtig zu laufen. Diese Erläuterungen brauchen wir später.

Die Stellung der Bürsten wird aus praktischen Gründen nicht von dieser Kurzschlußstellung aus gerechnet, sondern von einer davon $180^{\circ}$ entfernten. Von dieser aus gezählt, stehen die Bürsten also in der „Kurzschlußstellung“, wenn sie um $I 80^{\circ}$ verschoben sind.

In ihr muß ein richtig gebauter Motor die größte Stromaufnahme besitzen. Dies tut er dann, wenn der Stator die magnetomotorische Wirkung des Rotors aufhebt.

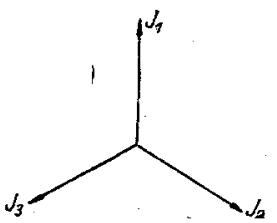

Fig. 6.
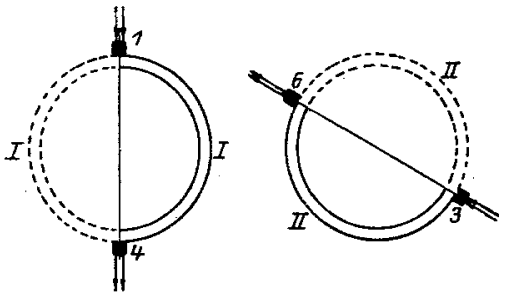

Fig. 7 .

Die magnetomotorische Wirkung des Rotors ist in den Fig. $6-8$ dargestellt. Fig. 6 ist das Zeitdiagramm der 3 Bürstenströme $\mathrm{J}_{1}, \mathrm{~J}_{2} \mathrm{~J}_{3}$, wir legen diesen Strömen den Höchstwert 2 bei.

Fig. 7 zeigt die Stromverteilung im Anker in jeder Phase, wie sie ausfallen würde, wenn der Strom jeder Phase im Höchstwert stände und allein vorhanden wäre. Jeder Bürstenstrom vom Werte 2 teilt sich, so daß in jeder der beiden Stablagen des Ankers Strom vom Werte $I$, dargestellt durch einfache Linien, fließt. 
Volle Linien bedeuten: der Strom fließt nach hinten (positive Richtung), gestrichelte bedeuten: Strom fließt nach vorn (negative Richtung).

Die Zeichen I, II, III deuten die Phase der Ströme an, z. B. sagt das Zeichen I aus: der Strom hat dieselbe Phase wie $J_{1}$.

Fig. 8 stellt das Resultat der Zusammenlegung der 3 Einzelbilder 7 dar. Man findet es. durch geometrische Summierung der 3 Einzelströme, die gemäß Fig. 7 in jeder Lage

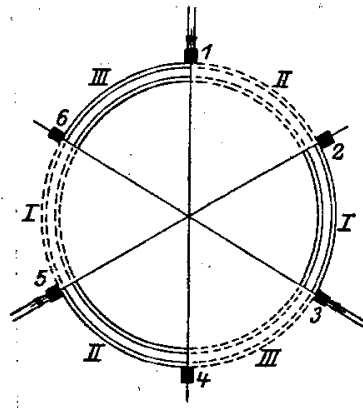

Fig. 8 . und jedem Sechstel des Ankerumfangs gedacht waren. Z. B. findet man für die obere Stablage des Sechstels rechts neben der Bürste I:

$$
\hat{+} \frac{\mathrm{I}}{2} \mathrm{~J}_{1} \stackrel{\wedge}{2} \mathrm{~J}_{2} \hat{+} \frac{\mathrm{I}}{2} \mathrm{~J}_{3}=\frac{\wedge}{\mathrm{J}_{2}}
$$

In Wirklichkeit fließen also in der unteren und oberen Stablage jedes Sechstels Ströme vom Werte und von der Phase der Bürstenströme, also je vom Werte 2, zusammen vom Werte 4. In der Fig. 8 ist dies durch vierfache Linien dargestellt.

Um den Anker möglichst zu kompensieren, muß der Stator gerade die entgegengesetzte Stromverteilung aufweisen. Seir. Umfang ist also mit Hilfe der Bürstenströme so zu speisen, daß der gesamte Strombetrag (Durchflutung) den Wert 4 bekommt. Daher muß der Stator gerade soviel Leiter erhalten, als der Anker besitzt, und diese müssen von den Bürstenströmen durchflossen werden.

Fig. 8 gibt nun an, daß z. B. der Strom der Bürste $I$ dem linken und rechten Sechstel des Stators zuzuführen ist, und zwar dem linken in positiver, dem rechten in negativer Richtung.

b) Die Selbsterregung mit Gleichstrom bei Verschiebung eines Bürstensatzes. Zur Untersuchung unserer Aufgabe benötigen wir nach diesen Feststellungen den Drehstrom nicht mehr. Wir nehmen jetzt gemäß Fig. 4 an, daß derselbe Strom bei den Bürsten I, 3, 5 eintritt, und verschieben die Bürsten 2, 4, 6, bei denen er austritt, so, daß sie von der Zählstelle aus um $\Upsilon_{5} 0^{\circ}$ verschoben erscheinen $\left(=30^{\circ}\right.$ aus der Kurzschlußstellung). Wir wissen, daß der Anker, wenn er bei dieser Bürstenstellung mit Drehstrom gespeist würde, im Sinne des Uhrzeigers als Motor umlaufen würde. Vorläufig nehmen wir aber an, daß der motorische Drehstrom fehlt und dieselbe Rotation durch einen äußeren Antrieb erhalten wird.

Der Strom, der bei I, 3, 5 eintritt, sei mit J bezeichnet und besitze den Wert 2. Die Unterscheidung der 3 Phasen haben wir nicht mehr nötig. Volle Linien sollen auch jetzt noch die positive (nach hinten), gestrichelte die negative (nach vorn) Raumrichtung des Stromes angeben, und zwar jede einfache Linie den Stromwert I.
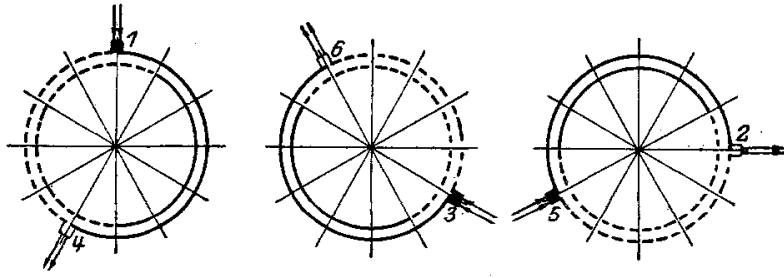

Fig. 9.

Fig. 9 stellt wieder den Stromverlauf dar, wie er sein würde, wenn nur in je einer Leitung Strom vorhanden wäre, z. B. nur in dem Kreise der Bürsten I-4. Legt man die 3 Einzelbilder 9 übereinander und addiert, in diesem Falle algebraisch, die Ströme jeder Lage, so findet man die resultierende Strom-

verteilung im Anker. Diese ist in Fig. Io innen wiedergegeben. Fig. II zeigt die wirkliche Stromverteilung der inneren und äußeren Stablage des Ankers.

Wie bei Fig. 8 besprochen wurde, ist der Stator so gewickelt und angeschlossen, $\mathrm{da} ß$ der in die Bürste I eintretende Strom zuvor das rechte und linke Sechstel der Statorwicklung durchströmt, und zwar so, daß er im rechten Sechstel die negative Richtung hat 
In Fig. ro muß das ebenso sein, und da die 3 Zweige sich alle gleichen, so ergibt sich die in Fig. Io außen wiedergegebene Stromverteilung im Stator.

Der Anker besitzt also abwechselnd Stellen von je $1 / 12$ Umfang mit den Durchflutungen $+2,0,-2,0,+2$ usw., der Stator Stellen von "je $1 / 6{ }^{\circ} \mathrm{Um}$ fang mit den Durchflutungen +4 und -4 .

In Fig. I2 ist der ganze Umfang abgewickelt und in der Treppenlinie D-D die gesamte Durchflutung dargestellt, die die Werte +4 , $+6,-4,-6,+4$ usw. besitzt.

Sie erzeugt am Umfang demnach 6 Pole, mit einer

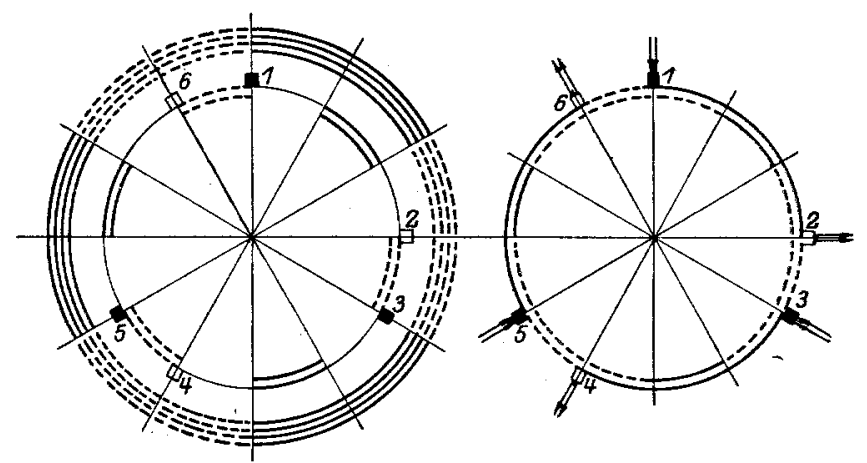

Fig, Io.

Fig. I I. dreieckartigen Flußverteilung, die in Fig. I2 durch die Linie F-F wiedergegeben ist.

Diese Flüsse bringen bei der angenommenen Drehungsrichtung im Uhrzeigersinne in sämtlichen Leitern elektromotorische Kräfte hervor.

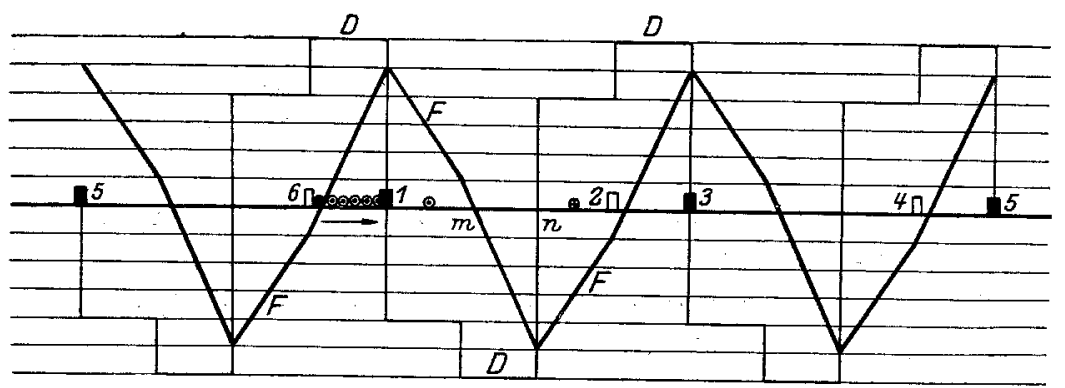

Fig. I2.

Wie Fig. I2 erkennen läßt, treten bei der Bürste I Kraftlinien aus dem Anker nach dem Stator hinüber. Austretende Linien seien positiv gezählt.

Ebenso treten im Gebiete zwischen den Bürsten 6 und I, zwischen denen die Ströme nach vorn fließen, Linien aus dem Anker aus. Die Leiter rotieren in der Richtung von 6 nach I hin. Nach bekannten Grundsätzen ist dann die EMK dieser Leiter ebenfalls nach vorn gerichtet. In Fig. I2 ist dies für einige Leiter eingetragen.

Wir erkennen also:

„Die von uns angenommene Stromrichtung wird durch die von ihr selbst erzeugten elektromotorischen Kräfte aufrecht erhalten. Ein Drehstromreihenschlußmotor von der Schaltung der Fig. 5 und der Bürstenstellung nach Fig. 9 erregt sich daher, während er als Motór vom Netzstrom getrieben läuft, selbst und überlagert dem Netzstrom einen Gleichstrom, der so verläuft, wie es Fig. I3 angibt.“

Fig. I2 zeigt auch, daß sich die EMK derjenigen Stellen gegenseitig aufheben, an denen sich die Ströme in der Ober- und Unterlage der Stäbe tilgen. So enthalten z. B. die Gebiete I bis $m$ und $\mathrm{n}$ bis 2 EMKe verschiedener Richtung. Nur rund die Hälfte aller Ankerleiter wird wirksam induziert. Dieser Umstand bringt es mit sich,

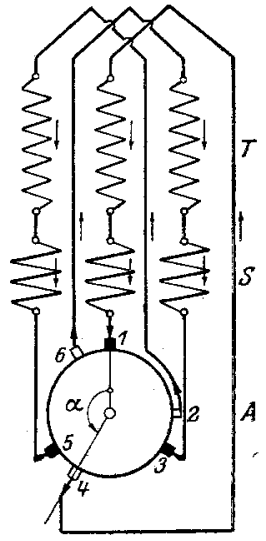

Fig. 13. daß das Ansprechen dieser Selbsterregung bereits mit einem verhältnismäßig kleinen OhmschenWiderstandunterdrückt werden kann, wie später ziffernmäßig nachgewiesen werden soll. 
Werden die verschiebbaren Bürsten $2,4,6$ um $30^{\circ}$ aus der Anfangsstellung ( $={ }_{5} 0^{\circ}$ aus der Kurzschlußstellung) verschoben, so ist die Bürstenverteilung ganz genau dieselbe wie bei der Verschiebung um $I_{5} 0^{\circ}$. Mithin ist auch hier Selbsterregung möglich.
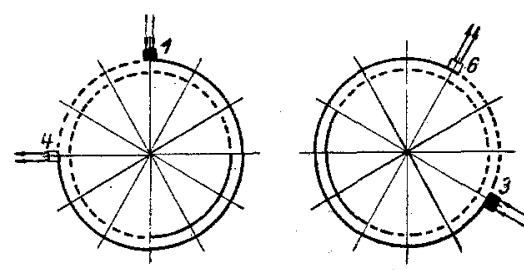

Fig. I 4 .

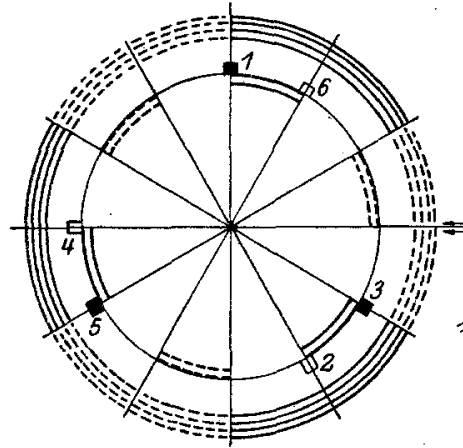

Fig. I 5 .

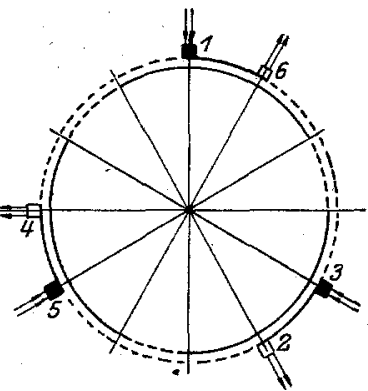

Fig. 16

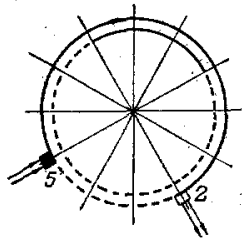

In der Mittelstellung zwischen beiden Fällen, also bei $90^{\circ} \mathrm{Ver}$ schiebung, liegt der Fall jedoch anders. In Fig. I4, I5, I6 und $I_{7}$ sind hierfür die den Fig. 9, Io, II und I2 entsprechenden Stromund Flußverteilungen gezeichnet. Zwischen Bürste I und 6 gehen die Ströme nach hinten, das Feld tritt a's dem Anker heraus, die Leiter bewegen sich von I nach 6, die EMKe sind daher nach vorn gerichtet.

Wir erkennen:

,Die von uns angenommenen Ströme werden bei der Bürstenverschiebung $90^{\circ}$ durch die von ihnen selbst erzeugten EMKe wieder vernichtet.

Ein Drehstrom-Reihenschluß. motor von der Schaltung nach

Fig. 5 und der Bürstenstellung nach Fig. I4 kann daher im Betriebe als Motor keine Selbsterregung aufweisen".

Fig. I7 läßt zugleich wieder erkennen, daß sich bei $90^{\circ}$ Verschiebung fast gerade ein Maximum der „Enterregung“ befindet.

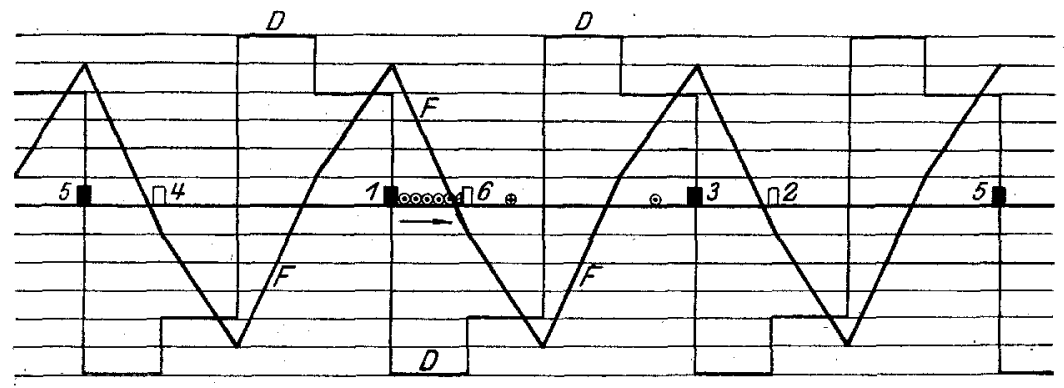

Fig. 17 .

Betrachten wir das ganze Bereich der Verschiebung, so erkennen wir also, daß von

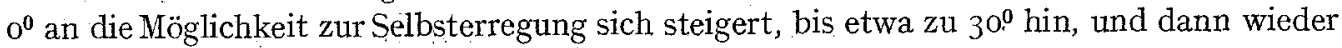
abnimmt. Bei $60^{\circ}$ Verschiebung ist sie Null. $Z$ wischen 60 und $100^{\circ}$ begegnen wir einem Gebiete, in dem im Gegensatz zum ersten Gebiete Neigung zur Enterregung besteht, mit einem Maximum bei $90^{\circ}$; endlich folgt noch ein Gebiet von $\mathrm{I}^{2} \mathrm{O}^{\circ}$ bis $180^{\circ}$ mit Neigung zur Selbsterregung, mit einem Höchstwert etwa bei $150^{\circ}$.

Die Figuren I2 und I7 zeigen, daß bei der größten Selbsterregung eine Induktionsspitze gerade am Orte der festen Bürsten I, 3, 5 liegt, während die Induktion bei den beweglichen Bürsten 2, 4, 6 nur klein ist. Die Selbsterregung ist daher mit erheblichem Feuer unter den festen Bürsten verbunden, während zu gleicher Zeit die beweglichen Bürsten funkenlos laufen können. 
c) Die Selbsterregung mit Gleichstrom bei Verschiebung aller Bürsten. Nicht immer werden die Drehstrom-Reihenschlußmotoren so ausgeführt, daß 3 Bürsten fest stehen bleiben, während 3 andere beweglich sind. Es kommt auch vor, daß die 6 Bürsten in gleichen Abständen stehen, wie in Fig. 5, und alle beweglich sind. Es ist leicht zu übersehen, daß in diesem Falle bei den Verschiebungen $0^{\circ}, 60^{\circ}, 120^{\circ}$ und $180^{\circ}$ die Neigung: zur Selbsterregung gleich Null ist, weil dann die Bürstenstellungen die gleichen sind wie vorher. Für die Verschiebung von $5_{50^{\circ}}$ gibt Fig. I8 die Strom- und Feldverteilungen an. Zwischen den Bürsten I und 6 gehen die Ströme nach vorn, das Feld tritt aus dem Anker heraus, die Leiter bewegen sich von 6 nach I, die EMKe sind daher nach vorn gerichtet. Mithin ist eine Selbsterregung möglich, bei der beide Bürstensätze gleich stark feuern.

Dasselbe gilt aus Symmetriegründen für die Verschiebung $30^{\circ}$, während für $90^{\circ}$ gemäß Fig. Ig sich wieder ein Maximum von Enterregung ergibt.

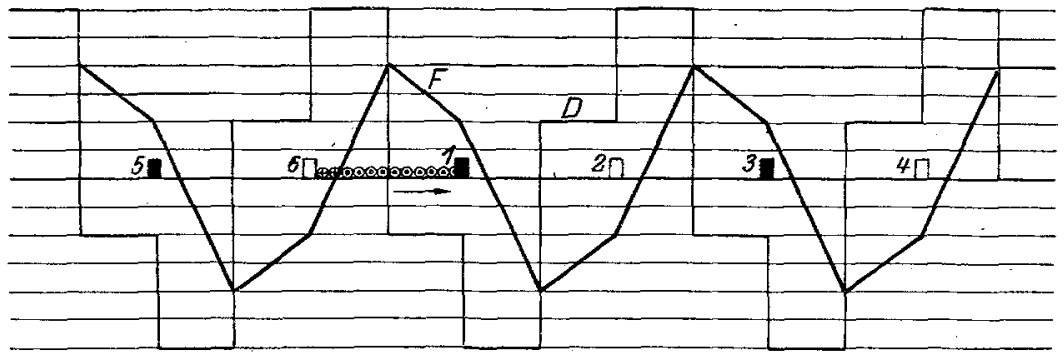

Fig. 18.

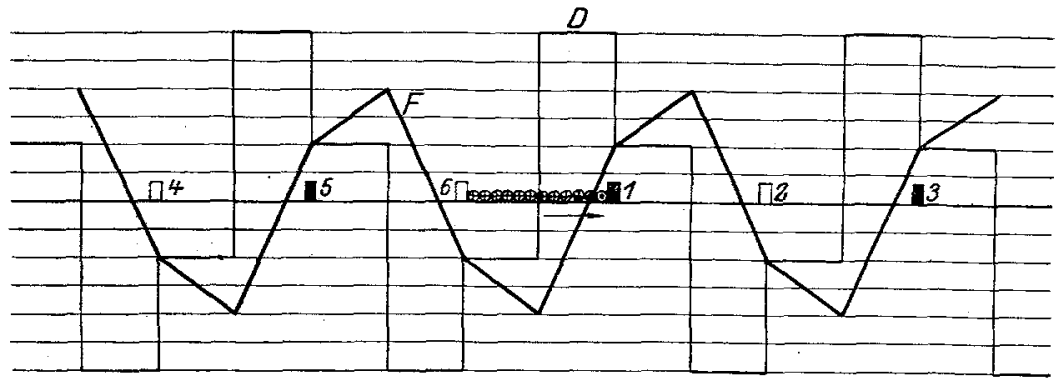

Fig. I9.

d) Vergleich zwischen $4 \mathrm{~b}$ und 4c. Ein Blick auf Fig. I2 und Vergleich mit Fig. I 8 lehrt, da $B$ der Motor mit einem festen und einem beweglichen Bürstensatz einen erheblichen Vorteil vor dem Motor mit nur beweglichen Bürsten voraus hat, den nämlich, daß bei ihm die Neigung zur Selbsterregung im Motorbetriebe viel geringer ist. Während nach Fig. 12 etwa die Hälfte aller Ankerleiter unwirksam bleibt, wie schon vorn gesagt, tragen in Fig. I8 beinahe alle Leiter zur Erregung bei.

Berücksichtigt man die verschiedenen Feldformen $\mathrm{F}-\mathrm{F}$ dieser Darstellungen, so verhält sich die EMK zwischen 2 Bürsten beim Motor mit festem und beweglichem Bürstensatz zur EMK zwischen 2 Bürsten beim Motor mit nur beweglichen Bürsten

wie $I: 2,166$.

Dieses Zahlenverhältnis läßt sich leicht aus den Bildern I2 und 18 herleiten.

5. Das Verhältnis der Selbsterregung zur Motorleistung. Um die Beschreibung der Selbsterregung zu vervollständigen, wollen wir noch ihre Flüsse und die 
elektromotorischen Kräfte mit denen des Motors selbst vergleichen. Hierzu müssen wir mitteilen, daß die Bürstenverschiebung bei einem voll belasteten Motor mit festem und beweglichem Bürstensatz meist $\mathbf{r} 20^{\circ}$ beträgt, wenn die festen Bürsten bei Null stehen. Wir nehmen an, daß der Motor dabei synchron laufe. Seine Rotorspannung ist dann Null. Die Spannung einer Statorphase gemessen in effektiven Volt sei $100 \%$, der Fluß, der diese Spannung erzeugt, gemessen in maximalen cgs, sei roo \% und die räumlich maximale Induktion, die dieses (Dreh-) Feld hat, sei auch mit $100 \%$ bezeichnet. Der Betriebsstrom, gemessen in effektiven Amp, sei roo \%. Wir denken uns nun durch äußere Widerstände - den Selbsterregungsgleichstrom auf den gleichen Amperewert eingestellt. Dann ergibt sich folgende Übersicht:

\begin{tabular}{|c|c|c|c|c|}
\hline \multirow[b]{2}{*}{. } & \multicolumn{2}{|c|}{ Spannung } & \multirow{2}{*}{$\begin{array}{l}\text { Fluß } \\
\%\end{array}$} & \multirow{2}{*}{$\begin{array}{l}\text { Max. } \\
\text { Induktion } \\
\%\end{array}$} \\
\hline & $\begin{array}{l}\text { gemessen } \\
\text { zwischen }\end{array}$ & $\begin{array}{c}\text { Wert } \\
\%\end{array}$ & & \\
\hline Motor normal betrieben . . & $\begin{array}{l}\text { z Stator- } \\
\text { klemmen } \\
\text { einer Phase }\end{array}$ & IOO & 100 & 100 \\
\hline $\begin{array}{l}\text { Motor selbst erregt, feste } \\
\text { und bewegliche Bürsten, } \\
a=150^{\circ} \ldots \ldots \ldots\end{array}$ & $\begin{array}{c}2 \text { be- } \\
\text { nachbarten } \\
\text { Bürsten }\end{array}$ & 20,2 & $3^{6,9}$ & 125 \\
\hline $\begin{array}{l}\text { Motor selbst erregt, nur be- } \\
\text { wegl. Bürsten, } \alpha=150^{\circ}\end{array}$ & $\begin{array}{l}2 \text { be- } \\
\text { nachbarten } \\
\text { Bürsten }\end{array}$ & 43,8 & 33,3 & 100 \\
\hline
\end{tabular}

6. Die Verhütung der Selbsterregung. Es ist nachgewiesen worden, daß der Drehstrom-Reihenschlußmotor Leistung. ins Netz zurückgeben kann, wenn durch einen Widerstand eine gleichzeitig mögliche Selbsterregung, die nicht mit der hier behandelten zu verwechseln ist, unterdrückt wird. In der Regel genügt dazu ein Widerstand, der $1 / 4$ der Motorleistung verbraucht, die Stromstärke von roo \% vorausgesetzt. Da nun nach der obigen Tabelle bei dem günstigeren der beiden Motoren nur $20 \%$ der Spannung auftreten, so genügt schätzungsweise zur Unterbindung der hier behandelten Selbsterregung beim Motorbetrieb ein Widerstand, der $1 / 4 \cdot 1 / 5=1 / 20$ oder $5 \%$ der Motorleistung verbraucht. Bei vielen Maschinen macht der Übergangswiderstand der Bürsten und der Widerstand der Wicklungen bereits so viel aus, daß ein äußerer Widerstand überhaupt entbehrlich wird. Es hat sich in der Tat an fertigen Maschinen sehr oft kein oder in den wenigen anderen Fällen nur ein ganz kleiner Widerstand als nötig erwiesen.

Die Maschine mit nur beweglichen Bürsten ist viel schlechter daran. Nach gleichen Gesichtspunkten beurteilt, muß der Widerstand hier für ca. II \% der Motorleistung bemessen sein, ein Widerstandswert, den die Maschine in ihrem Innern meistens nicht mehr besitzt, und der den Wirkungsgrad stark herabdrückt.

Diese Überschlagsrechnung über das nächstliegendste Verhinderungsmittel der Selbst. erregung im Motorbetriebe legt den Wunsch nahe nach Mitteln, die keine nennenswerten Verluste verursachen.

Ein derartiges Mittel scheint auch hier die Sättigung zu bieten, deren Wirkung nach den Forschungen der neuesten Zeit überhaupt die Möglichkeit zu verdanken ist, mit dem Drehstrom-Reihenschlußmotor Leistung in sein Netz zurückzugeben.

Ein weiteres Mittel bietet die Bürstenstellung. Wir sahen, daß die Neigung zur Selbsterregung Null ist bei den Bürstenverschiebungen 60 und $120^{\circ}$. Hierbei stehen jedesmal die beweglichen Bürsten mit den festen auf den gleichen Lamellen. Mithin genügt es, in den durch Selbsterregung gefährdeten Gebieten die bisher als fest angesehenen Bürsten auch $\mathrm{zu}$ verschieben und sie den anderen zu nähern.

Ein bequemeres und durchgreifendes Mittel bietet jedoch die Abtrennung des Rotors vom Stator durch den sog. Zwischentransformator unter Beachtung einer kieinen Vor- 
sichtsmaßregel. Man kann bekanntlich aus der Schaltung nach Fig. 5 oder I3 die Statorwicklung $\mathrm{S}$ entfernen und sie in den Kreis der -- dort nicht gezeichneten-Primärwicklung des Transformators T einfügen. Der Stromverlauf ist dann: Netz-Stator-Transformator primär-Transformator sekundär-Rotor.

Da Stator und Rotor immer zusammen die Selbsterregung liefern, nun aber durch den Transformator nur noch induktiv verbunden sind, ist an Stelle des Gleichstroms nur noch ein Wechselstrom denkbar. Momentan muß dieser freilich genau so fließen wie der bisher betrachtete Gleichstrom. Dies ist möglich, wenn je eine Stator- und eine Transformatorphase in Reihe, und diese 3 Reihen in Dreieck geschaltet sind. Der Selbsterregungsstrom durchläuft dann einfach den Umfang dieses Dreiecks bald in der einen, bald in der andern Richtung. Dagegen wird der Selbsterregungsstrom unterbunden, wenn die genannten 3 Reihen in Stern geschaltet sind. Denn dann würden die 3 Ströme in den 3 Reihen im gleichen Moment alle nach dem Sternpunkt hin fließen müssen, was ausgeschlossen ist.

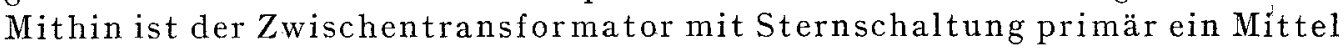
zur völligen Beseitigung dieser Ströme, während man die Dreieckschaltung besser nicht anwendet. Die (patentierte) für gewisse Betriebe vorteilhaft erscheinende SternDreieck-Umschaltung eines Motors mit Zwischentransformator ist deshalb mit Vorsicht zu gebrauchen.

Hiermit dürfte die bisher noch wenig bekannte, aber manches Interessante bietende Selbsterregungserscheinung des Drehstrom-Reihenschlußmotors im Betriebe als Motor genügend aufgeklärt sein. Sie ziffernmäßig weiterzuverfolgen, hat deshalb nicht soviel Wert, weil sie natürlich stets beseitigt werden $\mathrm{muB}$ und weil dies besonders bei dem Motor mit festem und beweglichem Bürstensatz leicht gelingt.

7. Schlußbemerkungen. Die Selbsterregung von Drehstrom-Reihenschluß-Motoren während des Betriebes als Motor wurde im Prüffelde der Siemens-Schuckertwerke zuerst im Januar IgI2 beim Leer-Anlassen eines solchen Motors beobachtet.

Wie aus einer an den Verfasser gerichteten Mitteilung vom 2r. Oktober Igr2 des Herrn Dipl.-Ing. Scherer (Österreichische Siemens-Schuckertwerke, Wien) hervorgeht, hat zuerst Herr Scher er bei einem Motor mit Zwischentransformator und Dreieckschaltung erkannt, daß die Selbsterregung einphasiger Wechselstrom sei, der im Motor die dreifache Polzahl ausbildet.

Unabhängig davon fand im November IgI2 im Prüffelde der Siemens-Schuckertwerke Herr Dr.-Ing. Dyhr, der mit der genaueren Untersuchung der Erscheinung beauftragt worden war, die Gleichstrom-Erregung des Motors mit Vordertransformator und die Unterdrückung der Erregung durch Zwischentransformator in Sternschaltung.

Wie endlich aus Patentanmeldungen der Firma Brown, Boveri \& Co. hervorgeht, wurde die Erscheinung dort ebenfalls beobachtet

\title{
Ein neues Verfahren zur harmonischen Analyse von Kurven.
}

\author{
Von \\ Rudoli Slaby.
}

1. Übersicht und Ziel. Fourier hat gezeigt, daß sich jede periodische Funktion durch eine Summe von Sinus- und Kosinusfunktionen darstellen läßt¹). Wir können schreiben:

1) Eine Zusammenstellung darauf bezüglicher Literatur bis zum Jahre I906 gibt Prof. E. Orlich in seinem Buche ,Aufnahme und Analyse von Wechseistromkurven", S. II4. In der ETZ finden sich folgende Veröffentlichungen: A. Fischer-Hinnen, I90I, S. 396; C. Runge, I905, S. 247; 